\title{
THE FORMATION OF BENZENE IN DENSE ENVIRONMENTS
}

\author{
P.M. Woods ${ }^{1}$
}

\begin{abstract}
PAHs are traditionally thought to form around carbon-rich AGB stars, where the presence of inner-wind shocks and nucleation seeds can aid their formation. However, my recent work has shown that the formation of benzene - thought to be the rate-limiting step in the formation of larger PAHs - can be efficient in other environments, namely the dense tori around the evolved stars of pre-planetary nebulae and in the inner regions of the accretion discs around young stars. I will discuss the chemical pathways to the formation of benzene in these regions and the subsequent formation of larger PAHs.
\end{abstract}

\section{Introduction}

The first discovery of benzene outside of the Solar System was made by Cernicharo et al. (2001a) towards the carbon-rich pre-planetary nebula ${ }^{2}$, CRL 618. A small absorption at $\sim 14.8 \mu \mathrm{m}$ in the mid-infrared spectrum from the Short-Wavelength Spectrometer on board the Infrared Space Observatory was attributed to the Q-branch of the $\nu_{4}$ band of benzene. These authors also detected lines of diacetylene $\left(\mathrm{C}_{4} \mathrm{H}_{2}\right)$ and triacetylene $\left(\mathrm{C}_{6} \mathrm{H}_{2}\right)$, and in the companion paper (Cernicharo et al. 2001b) detected methylpolyynes and other small hydrocarbons towards CRL 618. The detection of benzene was exciting for several reasons: firstly, this was another molecule to add to the list of 100 or so interstellar and circumstellar molecules detected at that time (the list has since grown to $\sim 160$ ). Secondly, benzene $\left(\mathrm{C}_{6} \mathrm{H}_{6}\right)$ is a large molecule, and very few molecules of this size have been detected in the interstellar or circumstellar media. Thirdly, benzene is a cyclic molecule, more circular in structure than the "triangular" cyclic molecules,

\footnotetext{
1 Jodrell Bank Centre for Astrophysics, School of Physics \& Astronomy, University of Manchester, Oxford Road, Manchester M13 9PL, UK

http://www.jb.man.ac.uk/ pwoods

2I use the term "pre-planetary nebula" as opposed to "protoplanetary nebula" (both terms describing evolved, post-Asymptotic Giant Branch stars) in order to save confusion with "protoplanetary disc", which is the accretion disc around a young, low-mass star.
} 
ethylene oxide $\left(\mathrm{c}-\mathrm{C}_{2} \mathrm{H}_{4} \mathrm{O}\right)$ and cyclopropenylidene $\left(\mathrm{c}-\mathrm{C}_{3} \mathrm{H}_{2}\right)$. As such, its detection heralded the beginnings of an astrochemistry (and an astrobiology) which was not solely dependent on the large linear carbon chain molecules previously seen in the archetypical carbon star, IRC +10216 , for example.

Five years subsequently, Bernard-Salas et al. (2006) reported the detection of benzene, diacetylene and triacetylene in another galaxy - the Large Magellanic Cloud. In this case, again, the object in question was a pre-planetary nebula, SMP LMC 11. Herpin \& Cernicharo (2000) suggested, for the case of CRL 618, that the molecular richness originates in a circumstellar torus which is sufficiently dense to shield molecules from the intense UV fields in the environments around these proto-White Dwarf stars. Certainly without sufficient shielding complex molecules in the post-AGB phase of evolution are rapidly destroyed (Woods et al. 2005). In these proceedings I will summarise work which shows that benzene can form efficiently in the post-AGB phase, in excellent agreement with the observations of Cernicharo et al. (2001a). I will also discuss the formation of benzene in a physically and chemically similar environment, the inner regions of protoplanetary discs, where benzene has yet to be detected but PAHs have been seen (Geers et al. 2006).

\section{Combustion chemistry or interstellar chemistry?}

Benzene and polycyclic aromatic hydrocarbons (PAHs) are readily found on Earth in crude oil and coal, and their combustion properties have been well-studied in the laboratory, motivated in part by the petroleum and combustion engine industries and latterly environmental concerns. Both benzene and PAHs are known to form in hydrocarbon flames under terrestrial conditions (e.g., Frenklach 2002), and could potentially form in circumstellar conditions where the chemistry and physics are similar. Such an approach has been used by combustion chemists (e.g., Frenklach \& Feigelson 1989) and astrochemists (e.g., Cau 2002; Cherchneff et al. 1992, see also Cherchneff, this volume) to explain carbon dust (soot) formation in the inner winds of carbon-rich red giant stars. However, the temperatures reached in flames and in the inner regions of AGB stars $(1000-1500 \mathrm{~K})$ are not readily found in the interstellar and much of the circumstellar media. Thus we must look to other explanations for the formation of benzene in pre-planetary nebulae, and given the chemical richness that some of these objects display, it seems logical to consider the "bottom-up" route to the formation of benzene and PAHs. (An alternative would be the "top-down" route, where graphitised carbon grains are decimated by shocks or other energetic processes).

Benzene has been detected in the upper atmospheres of Jupiter, Saturn and Titan (e.g., Bézard et al. 2001; Waite et al. 2007, 2005), environments where ion-molecule reactions dominate over the neutral-neutral reactions which are the mainstay of combustion chemistry. Similarly, if benzene were to form in the interstellar medium (ISM) or the intensely-irradiated environments around post-AGB stars, we would expect it to form via these ion-molecule reactions. Indeed, early 
models of dense interstellar clouds by McEwan et al. (1999) suggested that benzene was formed thus:

$$
\begin{array}{rll}
\mathrm{C}_{4} \mathrm{H}_{3}^{+}+\mathrm{C}_{2} \mathrm{H}_{2} & \longrightarrow c-\mathrm{C}_{6} \mathrm{H}_{5}^{+}+\mathrm{h} \nu \\
\left(\mathrm{C}_{4} \mathrm{H}_{3}^{+}+\mathrm{C}_{2} \mathrm{H}_{3}\right. & \left.\longrightarrow c-\mathrm{C}_{6} \mathrm{H}_{5}^{+}+\mathrm{H}\right) \\
c-\mathrm{C}_{6} \mathrm{H}_{5}^{+}+\mathrm{H}_{2} & \longrightarrow c-\mathrm{C}_{6} \mathrm{H}_{7}^{+}+\mathrm{h} \nu \\
c-\mathrm{C}_{6} \mathrm{H}_{7}^{+}+\mathrm{e}^{-} & \longrightarrow c-\mathrm{C}_{6} \mathrm{H}_{6}+\mathrm{H} .
\end{array}
$$

This model produced a fractional abundance of benzene of $\approx 10^{-9}$, several orders of magnitude below that detected in CRL 618. In order, then, to explain the observed abundance and investigate the formation of benzene in CRL 618, we will adopt an interstellar-based chemistry (Le Teuff et al. 2000; Millar et al. 2000), and a suitable physical model.

\section{The formation of benzene in pre-planetary nebulae}

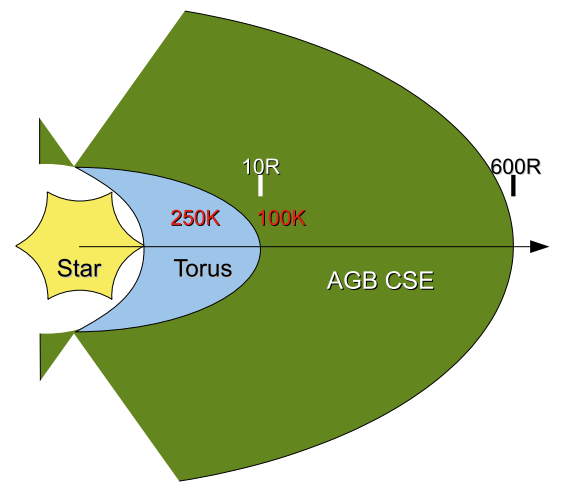

(a) From Herpin \& Cernicharo (2000)

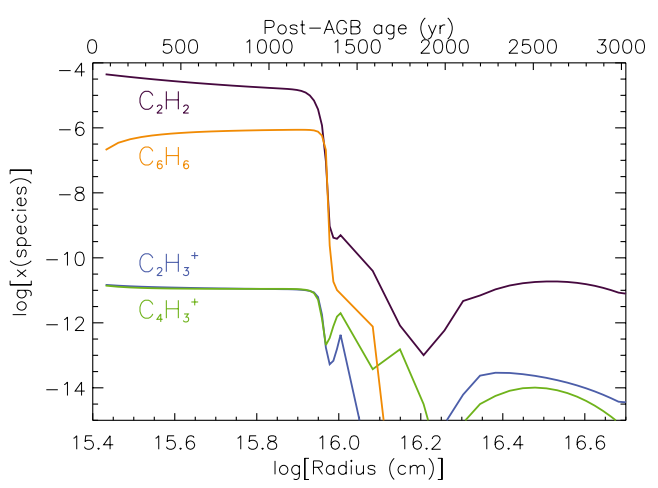

(b) Fractional abundance of $\mathrm{C}_{6} \mathrm{H}_{6} \&$ progenitors.

Fig. 1. Physical and chemical models of CRL 618.

Herpin \& Cernicharo (2000) were able to determine various physical properties of the circumstellar regions around CRL 618, and some of these are shown in Figure 1a. We choose to model the slowly-expanding $\left(5 \mathrm{~km} \mathrm{~s}^{-1}\right)$ torus, since the high density of this region ( $n \sim 10^{11} \mathrm{~cm}^{-3}$, initially) extinguishes the significant UV radiation $\left(2 \times 10^{5} \mathrm{G}_{0}\right)$ from the central star and allows complex chemistry to develop. We also enhance the cosmic-ray ionisation rate by a factor of 100 in order to account for X-rays from the hot star. At this stage of evolution, mass-loss from the star has ceased, and so we must consider a time-dependent situation. In the thin-shell approximation, we obtain the results shown in Figure 1b.

Benzene in the model is approximately 40 times less abundant than acetylene (a ratio observed by Cernicharo et al. 2001a) until about $1300 \mathrm{yr}$. At this stage, 
dilution of the expanding torus means that photodestruction of the molecular matter dominates. The major route to the formation of benzene in this environment is similar to that described in Section 2, except that the route to $\mathrm{C}_{4} \mathrm{H}_{3}^{+}$is simpler, viz.,

$$
\begin{aligned}
\mathrm{HCO}^{+}+\mathrm{C}_{2} \mathrm{H}_{2} & \longrightarrow \mathrm{C}_{2} \mathrm{H}_{3}^{+}+\mathrm{CO} \\
\mathrm{C}_{2} \mathrm{H}_{3}^{+}+\mathrm{C}_{2} \mathrm{H}_{2} & \longrightarrow \mathrm{C}_{4} \mathrm{H}_{3}^{+}+\mathrm{H}_{2} \\
\mathrm{C}_{2} \mathrm{H}_{2}^{+}+\mathrm{C}_{2} \mathrm{H}_{2} & \longrightarrow \mathrm{C}_{4} \mathrm{H}_{3}^{+}+\mathrm{H}
\end{aligned}
$$

(cf., McEwan et al. 1999). This is due to large fractional abundances of $\mathrm{HCO}^{+}$ (which is generally not abundant in AGB star envelopes but is abundant in the post-AGB phase because of the more intense UV flux from the star) and $\mathrm{C}_{2} \mathrm{H}_{2}$ (which is generally not abundant in the ISM). Combined with a high degree of UV-shielding and reasonable residence times, large amounts of benzene can form. For further details, see Woods et al. (2003, 2002).

\section{The formation of benzene in protoplanetary discs}

The inner regions of the protoplanetary accretion discs around young stars have a similar temperature and density to the torus modelled in the previous section. In this case, the physical model we use is significantly more complex (see Woods \& Willacy 2009, for a complete description) but the chemistry is very similar, although we use solar, oxygen-rich initial abundances, and incorporate gas-grain interactions and grain-surface reactions into the chemical modelling.

Results of the model show that benzene can form at a reasonably high fractional abundance $\left(\sim 10^{-6} \mathrm{~cm}^{-3}\right)$ in the inner $3 \mathrm{AU}$ of a protoplanetary disc. This is surprising, since protoplanetary discs are not carbon-rich environments; however, the cool midplanes of these discs allow water vapour to freeze out onto the surface of dust grains, effectively removing a major repository of oxygen from the gasphase chemistry. Small hydrocarbons can then dominate the chemistry until the advecting material is sufficiently warmed to liberate its oxygenous icy mantles. Interestingly, benzene forms in this environment via an odd-carbon-atom pathway rather than through the even-carbon-atom pathway (i.e., acetylene addition) discussed previously:

$$
\begin{array}{ccc}
\mathrm{C}_{3} \mathrm{H}_{4}+\mathrm{C}_{3} \mathrm{H}_{4}^{+} & \longrightarrow c-\mathrm{C}_{6} \mathrm{H}_{7}^{+}+\mathrm{H} \\
c-\mathrm{C}_{6} \mathrm{H}_{7}^{+}+\text {grain } & \longrightarrow g-\mathrm{C}_{6} \mathrm{H}_{6}+g-\mathrm{H} .
\end{array}
$$

and this ion-molecule pathway is more efficient than a suggested three-body neutralneutral reaction:

$$
\mathrm{C}_{3} \mathrm{H}_{3}+\mathrm{C}_{3} \mathrm{H}_{3}+\mathrm{M} \longrightarrow c-\mathrm{C}_{6} \mathrm{H}_{6}+\mathrm{M}
$$

The abundance of benzene in the gas phase depends on the binding energy of benzene to the grain surface on which it resides after neutralisation (reaction 4.2). 


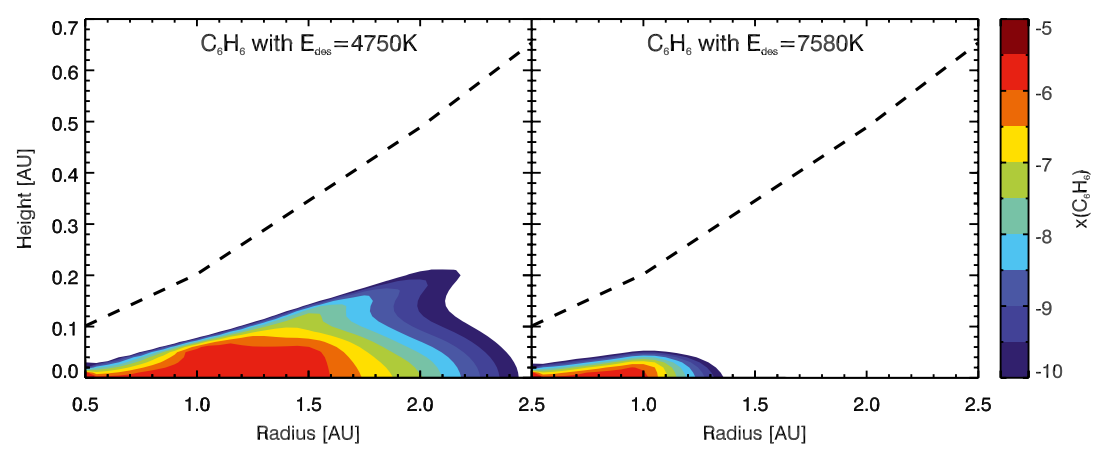

Fig. 2. The fractional abundance of gas-phase benzene in protoplanetary discs, using a low (left) and high (right) value of the binding energy. Dashed lines show the approximate disc surface.

Estimates of this binding energy vary, and thus two extreme cases $(4750 \mathrm{~K}$ and $7580 \mathrm{~K}$ ) are shown in Figure 2 for the distribution of gas-phase benzene. A recent experimental result suggests a value of $5600 \mathrm{~K}$ (Thrower et al. 2009) is appropriate, similar to the binding energy of water. The reaction between $\mathrm{C}_{3} \mathrm{H}_{4}$ and its ion (reaction 4.1) has been measured in the laboratory, and has been shown to occur for the main isomers of $\mathrm{C}_{3} \mathrm{H}_{4}$. These results are discussed in more detail in Woods \& Willacy (2007).

\section{The formation of PAHs in dense environments}

The formation of benzene is thought to be the rate-limiting step in the formation of PAHs, and there is evidence to support this (Wang \& Frenklach 1994). Potentially, then, once benzene is formed, PAHs could rapidly form from it, or from related species like phenyl. Second-ring closure (e.g., to form naphthalene from ethylbenzene or similar) is thought to involve an energy barrier which requires temperatures of 900-1100 K to overcome (Frenklach \& Feigelson 1989). Temperatures like this are present in the upper regions of protoplanetary discs, and material from the midplane of these discs is mixed to the surface on a timescale of $\sim 625 \mathrm{yr}$ (at 3 AU; Ilgner \& Nelson 2006). Vertical mixing is neglected in the model of Woods \& Willacy (2007), but we can estimate a formation timescale of a generic PAH (say with 40 carbon atoms - a size which is stable to photodissociation) from benzene. Two-carbon (acetylene) units can be added at a rate of one every three years, assuming a density of $\sim 10^{10} \mathrm{~cm}^{-3}$ and a conservative association rate. Adding 17 of these units to form a stable PAH would take $50 \mathrm{yr}$, significantly shorter than the mixing timescale calculated by Ilgner \& Nelson (2006). Thus it seems reasonable that PAHs could form in protoplanetary discs. Of course, a more detailed treatment is needed, and work is in progress on this topic.

Models of hydrocarbon chemistry in dense environments such as those discussed show that there is a disposition towards the formation of complex species, 
including benzene and PAHs. It may be that the destruction mechanisms of these complex molecules are not fully understood, but even if the carbon skeletons of molecules are shattered by some energetic process, the fragments themselves are the constituent building-blocks of PAHs, and can be re-incorporated into the formation process (Ní Chuimín 2009).

PMW would like to thank Tom Millar and Karen Willacy, who have contributed to this work over the past few years.

\section{References}

Bernard-Salas, J., Peeters, E., Sloan, G.C., et al., 2006, ApJ, 652, L29

Bézard, B., Drossart, P., Encrenaz, T., \& Feuchtgruber, H., 2001, Icarus, 154, 492

Cau, P., 2002, A\&A, 392, 203

Cernicharo, J., Heras, A.M., Tielens, A.G.G.M., et al., 2001a, ApJ, 546, L123

Cernicharo, J., Heras, A.M., Pardo, J.R., et al., 2001b, ApJ, 546, L127

Cherchneff, I., Barker, J.R., \& Tielens, A.G.G.M., 1992, ApJ, 401, 269

Frenklach, M., 2002, Phys. Chem. Chem. Phys., 4, 2028

Frenklach, M., \& Feigelson, E.D., 1989, ApJ, 341, 372

Geers, V.C., Augereau, J.-C., Pontoppidan, K.M., et al., 2006, A\&A, 459, 545

Herpin, F., \& Cernicharo, J., 2000, ApJ, 530, L129

Ilgner, M., \& Nelson, R.P., 2006, A\&A, 445, 223

Le Teuff, Y.H., Millar, T.J., \& Markwick, A.J., 2000, A\&AS, 146, 157

McEwan, M.J., Scott, G.B.I., Adams, N.G., et al., 1999, ApJ, 513, 287

Millar, T.J., Herbst, E., \& Bettens, R.P.A., 2000, MNRAS, 316, 195

Ní Chuimín, R., 2009, Ph.D. Thesis, Univ. of Manchester

Thrower, J.D., Collings, M.P., Rutten, F.J.M., \& McCoustra, M.R.S., 2009, MNRAS, 394, 1510

Waite, J.H., Young, D.T., Cravens, T.E., et al., 2007, Science, 316, 870

Waite, J.H., Niemann, H., Yelle, R.V., et al., 2005, Science, 308, 982

Wang, H., \& Frenklach, M., 1994, J. Phys. Chem., 98, 11465

Woods, P.M., \& Willacy, K., 2009, ApJ, 693, 1360

Woods, P.M., \& Willacy, K., 2007, ApJ, 655, L49

Woods, P.M., Nyman, L.-A., Schöier, F.L., et al., 2005, A\&A, 429, 977

Woods, P.M., Millar, T.J., Herbst, E., \& Zijlstra, A.A., 2003, A\&A, 402, 189

Woods, P.M., Millar, T.J., Zijlstra, A.A., \& Herbst, E., 2002, ApJ, 574, L167 